\title{
A Task Analytic Process to Define Future Concepts in Aviation
}

\author{
Brian F. Gore ${ }^{1}$, \& Cynthia Wolter ${ }^{2}$ \\ ${ }^{1}$ NASA Ames Research Center, Moffett Field, CA 94035-0001 \\ ${ }^{2}$ San Jose State University/NASA Ames Research Center, Moffett Field, CA 94035 \\ \{Brian.F.Gore, Cynthia.Wolter\}@nasa.gov
}

\begin{abstract}
A necessary step when developing next generation systems is to understand the tasks that operators will perform. One NextGen concept under evaluation termed Single Pilot Operations (SPO) is designed to improve the efficiency of airline operations. One SPO concept includes a Pilot on Board (PoB), a Ground Station Operator (GSO), and automation. A number of procedural changes are likely to result when such changes in roles and responsibilities are undertaken. Automation is expected to relieve the PoB and GSO of some tasks (e.g. radio frequency changes, loading expected arrival information). A major difference in the SPO environment is the shift to communication-cued crosschecks (verbal / automated) rather than movement-cued crosschecks that occur in a shared cockpit. The current article highlights a task analytic process of the roles and responsibilities between a PoB, an approach-phase GSO, and automation.
\end{abstract}

Keywords. Task analysis, concept evaluation, single pilot operations.

\section{Introduction}

The current day flight deck operational environment consists of a two-person Captain/First Officer crew. The current NextGen guidance is to optimize the efficiency of operations where feasible while maintaining the safety that exists in current operations. A concept of operations to reduce the commercial cockpit from the current twopilot crew, to a single pilot termed Single Pilot Operations (SPO) has been suggested as an option to optimize the efficiency of the NAS operations. The SPO concept has been under study by researchers in the Flight Deck Display Research Laboratory (FDDRL) at the National Aeronautics and Space Administration's (NASA) Ames and Langley Research Centers (1). Transitioning from a two-pilot crew to a single pilot crew will undoubtedly require changes in operational procedures, crew coordination, use of automation, and in how the roles and responsibilities of the flight deck and ATC are conceptualized in order to maintain the high levels of safety expected of the US National Airspace System.

The NextGen SPO environment would modify current day operations by reducing the crew complement onboard from two pilots to one pilot. The ground control 
system would also need to be modified to account for some of the responsibilities that would no longer be in the cockpit, operations like cross checks. One SPO concept maintains that three entities would share in the safe transport of the aircraft; a Pilot on Board (PoB), a Ground Station Operator (GSO), and automation. In this environment, both the PoB and the GSO would be fully trained pilots capable of flying the aircraft alone if incapacitation of one pilot should occur. Possible roles and responsibilities of a PoB, an approach-phase GSO, and automation are explored following a brief explanation of the current day roles and responsibilities.

\subsection{Current Day Operations}

The traditional roles of the cockpit operators are defined as Captain and First Officer roles. The Captain is the main pilot of the aircraft and the one who remains ultimately responsible for the aircraft, its passengers, and the crew. The Captain sits in the left seat of the cockpit. The first officer is the second pilot of an aircraft. The first officer sits in the right-hand seat in the cockpit. One pilot is designated the "pilot flying" (PF) and the other the "pilot not flying" (PNF), or "pilot monitoring" (PM), alternating during each flight as necessary. Even when the first officer is the flying pilot, the captain is in command and has legal authority of the aircraft. The amount of time either pilot is in control of the aircraft is near equal in normal operations, as the PF designation is passed back-and-forth throughout any given flight. In typical day-today operations, the essential job tasks are distributed fairly equally but final decisions always remains with the Captain (pilot-in-command). Some have defined the shared roles in the cockpit as being Aviate, Navigate, Communicate, and Systems Management in a task management hierarchy (2).

\subsection{Single Pilot Operations (SPOs)}

In SPOs, it is entirely possible that three entities will be required to guide the safe transport of the aircraft. These three entities include a Pilot on Board (PoB), a Ground Station Operator (GSO), and automation. In a proposed SPO environment, both the $\mathrm{PoB}$ and the GSO would be fully trained pilots capable of flying the aircraft alone in the event that incapacitation of the either human pilot should occur. Pilot Flying and Pilot Not Flying designations would vary between the PoB and the GSO, with possible multiple mid-flight reassignments. Most settings and radio communications would remain solely PNF responsibilities. Current Captain-specific tasks would remain the same and would always fall to the PoB. Both human operators would continually monitor instruments and radio communications, as well as perform crosschecks when notified of a change via voice or automation, and verify that the environment is consistent with their internal schema.

The PoB and the GSO means that the crew is operating essentially as a "separated cockpit". Due to a "separated cockpit", automation will be playing a large role in notifying the PoB and GSO of any changes (radio frequency, altitude, heading, speed, altimeters, CDU inputs/executions, entering/exiting holds, approach mode, speed brake, landing gear, touchdown zone elevation) so that either could verify without undue radio congestion. Advancements in automation may also relieve the human 
operators of some tasks such as loading expected arrival information, getting ATIS, and setting altimeters. A major notable difference between the current day and the SPO environment is the shift to 'communication-cued' crosschecks (verbal or automated) rather than 'movement-cued' crosschecks that occur in a shared cockpit. Automation will need to account for these overt and covert characteristics associated with a human "good crew member". Automation that mimics the characteristics of a "good crew member" can lead to increased efficiencies; which in turn lead to increased spare capacity to deal with unforeseen events.

\subsection{Research Objectives}

The objective of this research was to validate and refine sets of tasks associated with likely SPO environments. These tasks are linked together in a string of both sequential and parallel nodes. These nodes represent networks that can then be used to analyze different scenarios and task assignments for their impact on workload, taskload, task bottlenecks, efficiency, and safety. Possessing such task analyses allows researchers to explore the degree to which the location of pilots (remote or co-located) impact the ability to of the crew to work as an effective, separated, two-person crew as compared to a co-located two-person crew.

In an empirical study, pairs of pilots were asked to complete simulated flight segments in each of two conditions: co-located, and remote (1). The pilots were purposely presented with a critical situation that required problem-solving; one in which the crew encountered severe weather during their flight and needed to divert to an alternate airport. Scenarios added complexity to the diversion task, such as the amount of fuel onboard to support planned or unplanned diversions and system failures such as antiskid that required the crew to recalculate landing weights and distances.

The co-located condition required that pilots work together in a two-person flight simulator, a scenario that corresponded to current-day conditions. The remote condition required that the right and left seats of the cockpit be placed in different rooms, a scenario that represented a SPO concept. The crew in the SPO condition was allowed to communicate freely, however they could not see each other, observe each others' body language or point to information like weather cells on the navigation display. The interaction of the crew would be impacted by this change to SPO and part of the current analysis was to identify how the tasks would change as a function of such SPO operations.

Review of the above-described study was used to generate a preliminary high-level task analysis of both current day and SPO environments and for specific scenario development. Finer level of detail and validation came from subsequent interviews and collaboration with subject matter experts (SMEs).

\subsection{Method}

A task analysis is the process whereby the tasks to safely fly the aircraft with automation are analyzed, documented and outlined (3). The task analysis is a methodology covering a range of techniques to describe, and in some cases evaluate, the hu- 
man-machine and human-human interaction in systems. It is often described as the study of what an operator (or team) is required to do in terms of actions or cognitive processes to achieve a specific system state. Typically, it is characterized by a hierarchical decomposition of how a goal-directed task is accomplished, including a detailed description of activities, task and element durations, task frequency, task allocation, task complexity, environmental conditions, necessary clothing and equipment, and any other unique factors involved in, or required for, one or more people to perform a given task (3).

One type of task analysis, the Cognitive Task Analysis (CTA) identifies all of the critical cognitive tasks that the operator is required to perform with the automation (4, 5). CTA is a family of methods and tools for gaining access to the mental processes that organize and give meaning to observable behavior. CTA methods describe the cognitive processes that underlie the performance of tasks and the cognitive skills needed to respond adeptly to complex situations. Knowledge is elicited through indepth interviews and observations about cognitive events, structures, or models. Often the people who provide this information are subject matter experts (SMEs) - people who have demonstrated high levels of skill and knowledge in the domain of interest (6). The CTA is a complement to traditional task analysis as it adds the capability for designing for the unanticipated by describing the constraints on behavior rather than solely describing the behavior. These approaches feed into a concept-verification phase, where the research concept is verified by a human-system engineer, and preparations are made to implement the results from the task analyses into a model form (7).

For the current research, we created task decompositions that included both a task analysis and a semi-structured CTA of four scenarios (described below) of a planned approach into Denver starting at 37000' ASL with the crew operating under: (1) current day rules, (2) SPO-rules. Each rule set was run in either (3) nominal approach to land, or (4) an off nominal condition requiring the dynamic re-planning of an alternate airport. The task network analyses are represented with time-sequence profiles, task decomposition spreadsheets, 4D profiles, and task network representations.

\subsection{Task Representation}

Due to the complexity of the operational domains, four representations at varying levels of fidelity (from high level to lower levels) were created to convey the details associated with each approach to land rule set. This breakdown was necessary given the complexity of the tasks and of the tasks that shifted from the well-established concept to a new concept of operations as is the case with the SPOs. These representations of the tasks include a time-sequence profile, task decomposition spreadsheets, $4 \mathrm{D}$ profiles, and task network model representation.

- Time-sequence profile (high level): We began by creating very high-level time/sequence based profile of both nominal and divert approaches to Denver. This is termed time-sequence based because the analysis is represented along a timeline as the aircraft approaches the landing point and was not broken out by specific op- 
erator roles; only the tasks that were required to safely land an aircraft were identified. This process allowed us to identify task groups (not operator-specific) associated with the approaches. The task groups that were identified and classified were then broken down into a finer level of detail (Figure 1).

- Task decomposition spreadsheet (low level): The task decomposition spreadsheet was created to describe each task and operator roles in a more detailed, organized, in-depth manner to illustrate the task flow and the operator responsibilities. This complex representation of the task network allowed for a more evolved understanding of both the malleable and rigid associations between tasks (Figure 2).

- 4D profile (mid level): The high level-task groups were decomposed into individual, operator-specific tasks, and organized based on position of the aircraft and its phase of flight. This profile enabled side-by-side comparisons of current day and SPO environments as well as in-flight significant event conditions (Figure 3).

- Task Network Representation (low level): A linear, pictorial representation in Powerpoint was used to visualize the task network and to identify trouble spots where there is an increased task load due to the proposed SPO environment. By creating validated task groups, we can more fluidly re-organize task orders for analysis based on a given scenario (Figure 4).

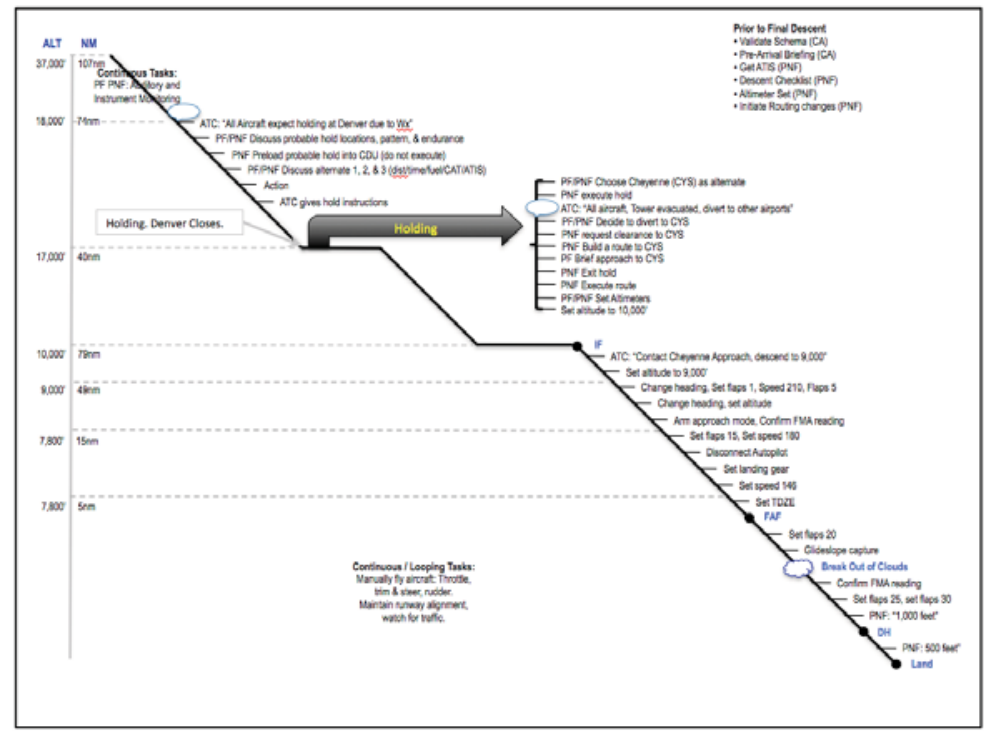

Fig. 1. Time-sequence profile 


\begin{tabular}{|c|c|c|c|c|c|c|}
\hline \multicolumn{7}{|c|}{ 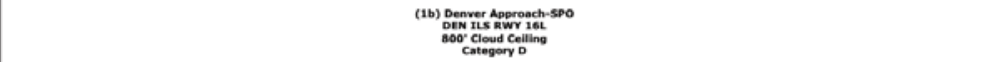 } \\
\hline Netitude & $\begin{array}{l}\text { Airport } \\
\text { Distance }\end{array}$ & 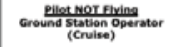 & 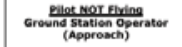 & 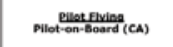 & Automation & ATC \\
\hline \multicolumn{2}{|c|}{ Prier to seenario Start: } & 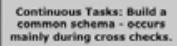 & 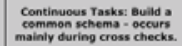 & 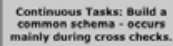 & & $\begin{array}{l}\text { Cantinuous taskes } \\
\text { Maintain separation }\end{array}$ \\
\hline \multicolumn{2}{|c|}{ Prior to Final Descent } & 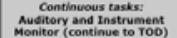 & 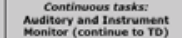 & 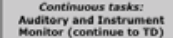 & & $\begin{array}{l}\text { Cantinuous tarks: } \\
\text { Maintain veparation }\end{array}$ \\
\hline & & & & & $\begin{array}{l}\text { Send night intomation to GSO } \\
\text { (Apoposoch) }\end{array}$ & \\
\hline & & 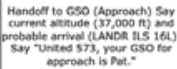 & connets & Listem, & & \\
\hline & & & 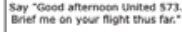 & & & \\
\hline & & Crosucteck. & Usten. Take notes. & 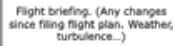 & & \\
\hline & & 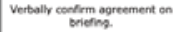 & usten. & usten. & & \\
\hline & & Dscromenter. & & & & \\
\hline & & & 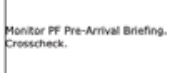 & 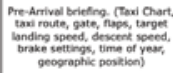 & & \\
\hline
\end{tabular}

Fig. 2. Task decomposition spreadsheet

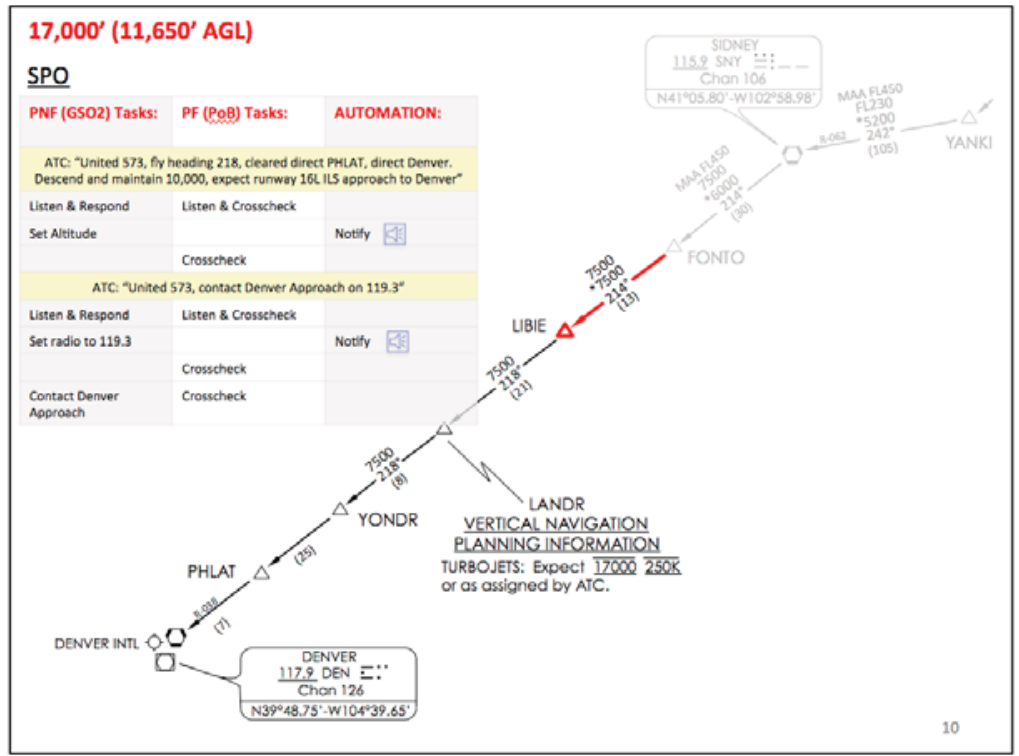

Fig. 3. 4D profile representation of the tasks 


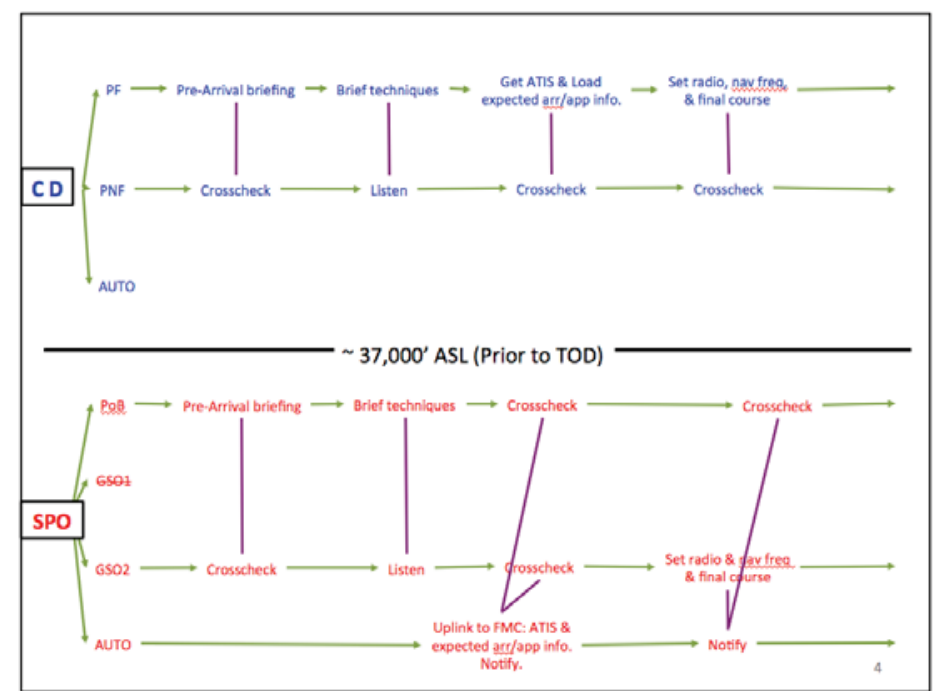

Fig. 4. Task network representation of the task analysis.

\subsection{Concept Verification Process and the Impact of SPO on Operator Roles and Responsibilities}

The task analyses were performed to determine the task differences between the current day and the proposed SPO descent and approach to land phases of flight, in addition to the changes in procedures when the crew is given divert commands from ATC regarding specific significant events (e.g. weather radar failure). Specific variables of interest included the role of communication, role of automation, role of crosschecks and its impact on crew coordination The analysis process began with a preexisting time / sequence-based profile of a descent into SFO, which was altered to represent the tasks required to descend into Denver, enter and exit a hold, decide to divert to Cheyenne, and to safely land the aircraft. This preliminary high-level representation of a significant event scenario was populated through direct observation of the SPO I study (1), SME evaluations and interviews, and published reports of anticipated NextGen tasks and operator errors $(8,9,10)$.

After final scenarios were chosen and populated with high-level tasks, they were refined and decomposed through the SPO concept reports and a series of SME interviews. Four spreadsheets of very detailed and ordered tasks representing each scenario were drafted and reviewed by SMEs (one current CA, and one former air traffic controller). The spreadsheets are organized by altitude, airport distance, operator tasks (PNF \& PF) with CA assignment, automation tasks, and ATC communications. Using the SME input, the task decomposition spreadsheet was modified to be more representative of the proposed SPO environment (11).

As per SME recommendation, 4D profiles were then created using the updated information from the spreadsheets (11). This representation specifies each task per- 
formed by the operators in the current day, and in the SPO environments. The tasks were overlaid onto the ownship's route, indicating the current phase of flight by including location, altitude, and nautical miles to destination. This profile assisted sideby-side comparisons of the differences between current day and SPO crew workload, highlighting high task-load phases of flight that could benefit from an increase in automated assistance. Using this information, tasks were restructured in the 4D profile representation, and both the time-sequence based profile and the task decomposition spreadsheet representations were edited to align with the changes.

All three representations went through a series of edits to create both an accurate representation of a current day environment, and a task distribution capable of representing a future SPO concept. A final SME interview was conducted to confirm the tasks and their orders illustrated in the representations and provide some further editing suggestions. All three representations created up to that point were refined further via the SME input, and the task networks began to be uploaded into Micro Saint Sharp (11).

The second SPO study was then observed to research future developments in the task analysis. Initial observations involved both the separated GSO/PoB and the colocated $\mathrm{CA} / \mathrm{FO}$ verbal and non-verbal communications through the headset and video monitors provided in the experimenter's control room. These observations also included participating in the crew debrief sessions and the GSO and PoB tools training sessions. This served to validate the task analyses already in progress and to provide context and direction for future analyses that more closely align with FDDRL studies.

\subsection{Candidate Roles and Responsibilities Considerations}

The preliminary evaluation separated the crewmembers to evaluate the kinds of interactions that could be expected when the crew was separated from each other, but need to coordinate. As a function of being separated, the crewmembers engaged in extra communications in order to insure that both separated crew members were operating according to a consistent mental map of the approach and the candidate divert options. These additional communications highlight a potential area of concern implementing SPO-like conditions; if the crew needs to take immediate action, they may be faced with few cognitive or attentional or even coordinated resources to safely land the aircraft as they are occupied getting to a consistent mental map. Alternatively, during the time period when the crew coordinates their activities, their attentional resources will be occupied to a greater extent than if they were already coordinated. This suggests that additional tasks cannot be added to the crew when in this situation, and it is only through a thorough analysis of the tasks that such bottlenecks can be identified. It is also important to highlight that SPO was an experimental simulation focused on examining a limited amount of the social interactions that exist between and among the crew. In the first SPO experiment, the crew was only separated and small changes were implemented in the roles for the crew to perform (the separated crew performed all of the tasks as if they were collocated with the PoB). It has been suggested however that one GSO may be responsible for multiple aircraft during nominal operations. The responsibility for multiple aircraft will change to the GSO 
being responsible for a single aircraft if the aircraft in question requires additional support or faces some other off-nominal kind of operation.

Transitioning between actively controlling multiple aircraft to actively controlling a single aircraft will be a challenge for the sector controller as well as the controller dedicating him/herself to the additional aircraft. Additional research is needed to further evaluate these conditions and the scenarios that were explored in a second SPO experiment in 2013.

\subsection{Future Research}

The SPO scenarios defined thus far represent two flight conditions and one potential way of assigning tasks between entities. Future effort will refine the existing task analysis based on additional SME evaluations, as well as extend the task analysis to additional divert locations to parallel ongoing HITL simulations being completed by the Flight Deck Display Research Laboratory (FDDRL). Modifications to the existing scenarios include manipulating the GSO to control multiple aircraft versus dedicated assistance requests, scenarios comparing different levels of automation (e.g. notification of pilot initiated changes, initiation of changes uplinked from ATC or automated, setting changes), scenarios with significant events other than/in addition to weather (e.g., cargo door open), and evaluate the impact on the number of tasks required of the current GSO, the new GSO and the interaction that needs to occur with the PoB. In the current SPO iterations, flight roles and responsibilities were primarily attended to by the PoB (CA) during cruise through the top of descent. The responsibilities of a typical current day FO are assumed by the GSO at that point and continue to touchdown (i.e., ATC communications, radio frequency settings, heading settings, one altimeter setting, altitude settings, flap settings). In future iterations, other role assignments may show a lessening of task load for both human operators. Analysis of the benefits if the roles of automation are expanded based on projected automation advancements available in the future. In addition, the GSO and PoB may be flexible with their roles and responsibilities and assign tasks differently for each flight based on flight conditions, emergency situations, and experience level. Task type as a function of the operator role under both current day and future SPO operations under additional divert conditions are also possible areas of research for the SPO environment. It is expected that thorough task decompositions of the various scenarios will provide insight into the impact of required and time critical flight crew and ATC tasks under SPO technologies and procedures. Methods that feed the understanding of the task environment such as human in the loop simulations will lead to more comprehensive understanding of the effects of such a conceptual change on the operations of a complex environment such as those as exemplified by SPO.

\subsection{References}

1. Johnson, W., Lachter, J., Feary, M., Comerford, D., Battiste, V., Mogford, R. Task Allocation For Single Pilot Operations: A Role For The Ground. In HCI Aero 2012 - Interna- 
tional Conference on Human-Computer International Aerospace; Brussels; 12-14 Sept, 2012. Belgium (2012)

2. Schutte. P. C., \& Trujillo, A. C. Flight Crew Task Management In Non-Normal Situations. Proceedings of the 40th Annual Meeting of the Human Factors and Ergonomics Society, 244-248. HFES, Santa Monica, CA (1996)

3. Kirwan B., Ainsworth L. K. A Guide To Task Analysis. Taylor \& Francis, London, UK (1992)

4. Diaper D. Task Analysis for Human-Computer Interaction. Ellis Horwood Limited, England (1989)

5. Zachary, W., Ryder, J., and Hicinbothom, J. Cognitive Task Analysis \& Modeling Of Decision Making In Complex Environments. In Salas, E. \& J. Cannon-Bowers (Eds.), Making Decisions Under Stress. American Psychological Association, Washington, DC (1998)

6. Klein, G. Cognitive Task Analysis Of Teams. In Schraagen, Chipman, \& Shalin, Eds. Cognitive Task Analysis, Lawrence Erlbaum Mahwah, NJ. 417-430. (2000)

7. Gore, B.F. Chapter 32: Human Performance: Evaluating The Cognitive Aspects. In V. Duffy (ed.), Handbook of Digital Human Modeling, pp.32-1-32-18. CRC Press/Taylor \& Francis, Boca Raton (2008)

8. Gore, B. F., Hooey, B. L., Mahlstedt, E. A., \& Foyle, D. C. Evaluating NextGen Closely Spaced Parallel Operations concepts with validated human performance models: Scenario development and results. (NASA TM-2013-216503). NASA Ames Research Center. Moffett Field, CA (2013)

9. Gore, B. F., Hooey, B. L., Haan, N. J., Socash, C., Mahlstedt, E. A., \& Foyle, D. C. A validated set of MIDAS v5 task network model scenarios to evaluate NextGen Closely Spaced Parallel Operations concepts. (HCSL 13-03). NASA Ames Research Center. Moffett Field, CA (2013)

10. Gore, B. F., Hooey, B. L., Haan, N., Bakowski, D. L., \& Mahlsted, E. A methodical approach for developing valid human performance models of flight deck operations. Paper presented at the 14th Annual HCI International 2011, Orlando, FL. (2011, July 9 - July 14)

11. Wolter, C.A. \& Gore, B.F. ASP/CTD/SPO Task Summary Report: Single Pilot Operations (SPO) ConOps-related gaps and research issues identified by analysis of SPO Pilot and Controller tasks, technologies and procedures. HCSL Technical Report (HCSL-13-07). Human Centered Systems Laboratory (HCSL), NASA Ames Research Center, Moffett Field, CA. (2013)

Acknowledgements. The composition of this work was supported by the Single Pilot Operations (SPO) project of NASA's Aeronautics Research Mission Directorate Task Number (NASA POC Dr. Walter Johnson). The authors would like to thank the SMEs Rob Kotesky, and Vern Battiste for their invaluable assistance identifying the likely SPO tasks, the entire SPO research staff from the FFDRL, and all reviewers for their insightful comments. 